Tôhoku Math. Journ.

30 (1978), 463-470.

\title{
WEIGHT FUNCTIONS ON PROBABILITY SPACES
}

\section{AKIHITo UChIYAMA}

(Received April 19, 1977)

Introduction. B. Muckenhoupt [3] proved that a nonnegative function $V \in L_{\text {loc }}^{1}(R)$ satisfies

(*) $\quad \sup \left\{\int_{R} f^{*}(x)^{p} V(x) d x\left(\int_{R}|f(x)|^{p} V(x) d x\right)^{-1}: f\right.$ is measurable $\}<\infty$, where $1<p<\infty$ and $f^{*}$ is the Hardy maximal function

$$
f^{*}(x)=\sup \left\{(y-x)^{-1} \int_{x}^{y}|f(t)| d t: y \in R \backslash\{x\}\right\},
$$

if and only if

$$
\begin{aligned}
A_{p}(V)= & \sup \left\{(y-x)^{-p} \int_{x}^{y} V(t) d t\left(\int_{x}^{y} V(t)^{-1 /(p-1)} d t\right)^{p-1}:\right. \\
& -\infty<x<y<\infty\}<\infty .
\end{aligned}
$$

The proof of this result consists of the following two theorems. [See also R. Coifman and C. Fefferman [1].]

THEOREM A. Let $U$ and $V$ be nonnegative measurable functions on $R, p \in(1, \infty)$,

$$
\begin{aligned}
A_{p}(U, V)= & \sup \left\{(y-x)^{-p} \int_{x}^{y} U(t) d t\left(\int_{x}^{y} V(t)^{-1 /(p-1)} d t\right)^{p-1}:\right. \\
& -\infty<x<y<\infty\}
\end{aligned}
$$

and

$$
\begin{gathered}
W_{p}(U, V)=\sup \left\{\lambda^{p} \int_{\left\{f^{*}>\lambda\right\}} U(t) d t\left(\int_{R}|f(t)|^{p} V(t) d t\right)^{-1}:\right. \\
\lambda>0, f \text { is measurable }\} .
\end{gathered}
$$

Then

$$
A_{p}(U, V) \leqq W_{p}(U, V) \leqq C(p) A_{p}(U, V)
$$

THEOREM B. If $V$ is a nonnegative measurable function and $A_{p}(V)<M$ for some $p \in(1, \infty)$ and $M<\infty$, then there exist $\delta(M, p)>0$ 
and $N(M, p)<\infty$ such that

$$
\begin{aligned}
H_{\delta}(V)= & \sup \left\{(y-x)^{\delta /(1+\delta)}\left(\int_{x}^{y} V(t)^{1+\delta} d t\right)^{1 /(1+\delta)}\left(\int_{x}^{y} V(t) d t\right)^{-1}:\right. \\
& -\infty<x<y<\infty\}<N .
\end{aligned}
$$

Since $A_{p /(p-1)}\left(V^{-1 /(p-1)}\right)=A_{p}(V)^{1 /(p-1)}$, applying Theorem $\mathrm{B}$ to $V^{-1 /(p-1)}$ we get

$$
A_{p-\varepsilon}(V) \leqq\left(A_{p /(p-1)}\left(V^{-1 /(p-1)}\right) H_{\varepsilon /(p-\varepsilon-1)}\left(V^{-1 /(p-1)}\right)\right)^{p-1}<\infty
$$

for some $\varepsilon>0$. Then $(*)$ follows from Theorem A and the Marcinkiewicz interpolation theorem. On the other hand, by Theorem A it is clear that (*) implies $A_{p}(V)<\infty$.

In this note we consider these theorems on a probability space with a sequence of nondecreasing sub $\sigma$-fields. The definitions of the maximal function, $A_{p}, W_{p}$, and $H_{\delta}$ on this probability space will be given in the following sections. The essential techniques are due to [1] and [3].

1. The "weak type" problem. Let $(\Omega, F, P)$ be a probability space with a sequence of sub $\sigma$-fields

$$
F_{1} \subset F_{2} \subset \cdots \subset F_{n} \subset \cdots \subset F
$$

such that $\bigvee_{n=1}^{\infty} F_{n}=F$. Let $V, U$, and $X$ be any nonnegative $F$-measurable functions. Let $\varepsilon$ and $\lambda$ be arbitrary positive numbers. We define $\circ, X^{*}, A_{p}$ and $W_{p}(1 \leqq p<\infty)$ as follows:

$$
\begin{aligned}
& a \circ b=a b \quad \text { for } a, b \in[0, \infty), \\
& a \circ \infty=\infty \circ a=\infty \quad \text { for } a \in[0, \infty], \\
& X^{*}=\sup _{n} E\left[X \mid F_{n}\right], \\
& A_{p}(U, V)=\sup _{n}\left\|E\left[V^{-1 /(p-1)} \mid F_{n}\right]^{p-1} E\left[U \mid F_{n}\right]\right\|_{\infty} \text { for } p \in(1, \infty), \\
& A_{1}(U, V)=\sup _{n}\left\|V^{-1} E\left[U \mid F_{n}\right]\right\|_{\infty}, \\
& W_{p}(U, V)=\sup _{X, \lambda} \lambda^{p} \int_{\left\{X^{*}>\lambda\right\}} U d P\left(\int X^{p} V d P\right)^{-1} \text { for } p \in[1, \infty) .
\end{aligned}
$$

The above definition of $A_{p}$ is due to M. Izumisawa and N. Kazamaki [2]. In the case $U=V$, they proved that $A_{p}<\infty$ implies

$$
\sup _{x} \int X^{* q} V d P\left(\int X^{q} V d P\right)^{-1}<\infty
$$

for $q>p$ and conversely 


$$
\sup _{X} \int X^{* p} V d P\left(\int X^{p} V d P\right)^{-1}<\infty
$$

implies $A_{p}<\infty$.

Following the theory of [1] and [3], we extend the result of [2], that is,

TheORem 1. $A_{p}=W_{p}$ for $p \in[1, \infty)$.

REMARK. The fact that $W_{p} \leqq A_{p}$ has been pointed out by T. Tsuchikura.

For the proof of Theorem 1 we prove the following three lemmas.

LEMMA 1. Set

$$
X^{* *}=\sup _{n} E\left[X V \mid F_{n}\right] \circ E\left[U \mid F_{n}\right]^{-1} .
$$

Then $\lambda \int_{\left\{X^{* *}>\lambda\right\}} U d P \leqq \int X V d P$.

Proof. Set

$$
\begin{aligned}
& B_{n}=\left\{\omega \in \Omega: E\left[X V \mid F_{n}\right] \circ E\left[U \mid F_{n}\right]^{-1}>\lambda\right. \text { and } \\
& \left.E\left[X V \mid F_{i}\right] \circ E\left[U \mid F_{i}\right]^{-1} \leqq \lambda \text { for } i=1,2, \cdots, n-1\right\} .
\end{aligned}
$$

Since $B_{n} \in F_{n}$,

$$
\lambda \int_{B_{n}} U d P=\lambda \int_{B_{n}} E\left[U \mid F_{n}\right] d P \leqq \int_{B_{n}} E\left[X V \mid F_{n}\right] d P=\int_{B_{n}} X V d P .
$$

Summing up for $n=1,2, \cdots$, we get the desired inequality.

Lemma 2. Let $F^{\prime}$ be an arbitrary sub $\sigma$-field of $F$. Then

$$
X \leqq \lim _{n \rightarrow \infty} E\left[X^{n} \mid F^{\prime}\right]^{1 / n} \quad \text { a.s. }
$$

Proof. By Hölder's inequality $E\left[X^{n} \mid F^{\prime}\right]^{1 / n}$ is monotone increasing. Set

$$
B_{\lambda}=\left\{\omega \in \Omega: \lim _{n \rightarrow \infty} E\left[X^{n} \mid F^{\prime}\right]^{1 / n} \leqq \lambda\right\} .
$$

Then it suffices to show that $X \leqq \lambda$ on $B_{\lambda}$. Since $B_{\lambda} \in F^{\prime}$,

$$
\begin{gathered}
(\lambda+\varepsilon)^{n} P\left(B_{\lambda} \cap\{\omega: X>\lambda+\varepsilon\}\right) \leqq \int_{B_{\lambda}} X^{n} d P \\
=\int_{B_{\lambda}} E\left[X^{n} \mid F^{\prime}\right] d P \leqq \lambda^{n} P\left(B_{\lambda}\right) .
\end{gathered}
$$

Thus letting $n \rightarrow \infty$, we have

$$
P\left(B_{\lambda} \cap\{\omega: X>\lambda+\varepsilon\}\right)=0
$$

and we get the desired inequality. 
LEMMA 3. $W_{p}=\lim _{\varepsilon \downarrow 0} W_{p+\varepsilon}$.

Proof. As it is trivial that $W_{p} \leqq \lim \inf _{\varepsilon \downarrow 0} W_{p+\varepsilon}$, it suffices to prove that $W_{p} \geqq \lim \sup _{\varepsilon \downarrow 0} W_{p+\varepsilon}$. Take an arbitrary $\alpha \in(0,1)$. Set $B_{\alpha \lambda}=$ $\{X>\alpha \lambda\}$. Then

$$
\begin{aligned}
\lambda^{p+\varepsilon} & \int_{\left\{X^{*}>\lambda\right\}} U d P\left(\int X^{p+\varepsilon} V d P\right)^{-1} \\
& \leqq \lambda^{\varepsilon} \lambda^{p} \int_{\left.\left(\left(I I B_{\alpha \lambda}\right) X\right)^{*}>(1-\alpha) \lambda\right\}} U d p\left(\lambda^{\varepsilon} \alpha^{\varepsilon} \int\left(I\left(B_{\alpha \lambda}\right) X\right)^{p} V d P\right)^{-1} \\
& =\alpha^{-\varepsilon}(1-\alpha)^{-p}(1-\alpha)^{p} \lambda^{p} \int_{\left(\left(I\left(B_{\alpha \lambda}\right) X\right)^{*}>(1-\alpha) \lambda\right\}} U d P\left(\int\left(I\left(B_{\alpha \lambda}\right) X\right)^{p} V d P\right)^{-1} \\
& \leqq \alpha^{-\varepsilon}(1-\alpha)^{-p} W_{p},
\end{aligned}
$$

where $I(B)$ is the indicator function of a measurable set $B$. Thus we get lim sup $\operatorname{su}_{\varepsilon \downarrow} W_{p+\varepsilon} \leqq(1-\alpha)^{-p} W_{p}$ for any $\alpha \in(0,1)$, i.e., $\lim \sup _{\varepsilon \downarrow 0} W_{p+\varepsilon} \leqq$ $W_{p}$.

Proof of Theorem 1. First we consider the case $1<p<\infty$. Since

$$
\begin{aligned}
E\left[X \mid F_{n}\right] & \leqq E\left[X^{p} V \mid F_{n}\right]^{1 / p} \circ E\left[V^{-1 /(p-1)} \mid F_{n}\right]^{(p-1) / p} \\
& \leqq\left(A_{p}+\varepsilon\right)^{1 / p}\left(E\left[X^{p} V \mid F_{n}\right] \circ E\left[U \mid F_{n}\right]^{-1}\right)^{1 / p},
\end{aligned}
$$

by Lemma 1

$$
\lambda^{p} \int_{\left(X^{*}>\lambda\right\}} U d P \leqq \lambda^{p} \int_{\left(\left(A_{p}+\varepsilon\right)\left(X^{p}\right) *>>\lambda p\right\}} U d P \leqq\left(A_{p}+\varepsilon\right) \int X^{p} V d P .
$$

Thus we get

$$
W_{p} \leqq A_{p} \text {. }
$$

Let $n$ be an arbitrary positive integer and $\alpha$ be an arbitrary number greater than 1. Set

$$
\begin{gathered}
B_{i j}=\left\{\omega: E\left[V^{-1 /(p-1)} \mid F_{n}\right] \in\left(\alpha^{i}, \alpha^{i+1}\right], E\left[U \mid F_{n}\right] \in\left(\alpha^{j}, \alpha^{j+1}\right]\right\}, \\
B_{i \infty}=\left\{\omega: E\left[V^{-1 /(p-1)} \mid F_{n}\right] \in\left(\alpha^{i}, \alpha^{i+1}\right], E\left[U \mid F_{n}\right]=\infty\right\}
\end{gathered}
$$

and

$$
B_{\infty}=\left\{\omega: E\left[V^{-1 /(p-1)} \mid F_{n}\right]=\infty\right\}
$$

for $i, j=0, \pm 1, \pm 2, \cdots$ Let $Y=V^{-1 /(p-1)} I\left(B_{i j}\right)$. By $B_{i j} \in F_{n}$,

$$
\begin{aligned}
& \alpha^{i p} \alpha^{j} P\left(B_{i j}\right) \leqq \alpha^{i p} \int_{B_{i j}} E\left[U \mid F_{n}\right] d P=\alpha^{i p} \int_{B_{i j}} U d P \\
& \quad \leqq \alpha^{i p} \int_{\left\{Y^{\left.*>\alpha^{i}\right\}}\right.} U d P \leqq\left(W_{p}+\varepsilon\right) \int Y^{p} V d P \leqq\left(W_{p}+\varepsilon\right) \int_{B_{i j}} V^{-1 /(p-1)} d P \\
& \quad=\left(W_{p}+\varepsilon\right) \int_{B_{i j}} E\left[V^{-1 /(p-1)} \mid F_{n}\right] d P \leqq\left(W_{p}+\varepsilon\right) \alpha^{i+1} P\left(B_{i j}\right) .
\end{aligned}
$$


Thus if $P\left(B_{i j}\right) \neq 0$,

$$
\left(\alpha^{-1} E\left[V^{-1 /(p-1)} \mid F_{n}\right]\right)^{p-1}\left(\alpha^{-1} E\left[U \mid F_{n}\right]\right) \leqq W_{p} \alpha
$$

a.s. on $B_{i j}$. Using the same argument for $Y=V^{-1 /(p-1)} I\left(B_{i \infty}\right)$ we get

$$
\alpha^{i p} \infty P\left(B_{i \infty}\right) \leqq\left(W_{p}+\varepsilon\right) \alpha^{i+1} P\left(B_{i \infty}\right), \quad \text { i.e., } \quad P\left(B_{i \infty}\right)=0 .
$$

Let $T_{j}=\min \left(V^{-1}, j\right)$ and $Y=T_{j}^{1 /(p-1)} E\left[T_{j}^{1 /(p-1)} \mid F_{n}\right]^{-1 / p}$. Then

$$
\begin{aligned}
& \lambda^{p} \int_{\left\{E\left[T_{j}^{1 /(p-1)} \mid F_{n}\right]>\lambda p /(p-1)_{\}}\right.} U d P \leqq \lambda^{p} \int_{\left\{Y^{*}>\lambda\right\}} U d P \leqq\left(W_{p}+\varepsilon\right) \int Y^{p} V d P \\
& \quad=\left(W_{p}+\varepsilon\right) \int E\left[T_{j}^{p /(p-1)} V \mid F_{n}\right] E\left[T_{j}^{1 /(p-1)} \mid F_{n}\right]^{-1} d P \\
& \quad \leqq\left(W_{p}+\varepsilon\right) \int E\left[T_{j}^{1 /(p-1)} \mid F_{n}\right] E\left[T_{j}^{1 /(p-1)} \mid F_{n}\right]^{-1} d P \leqq W_{p}+\varepsilon .
\end{aligned}
$$

Letting $j \rightarrow \infty$, we get

$$
\lambda^{p} \int_{B_{\infty}} U d P \leqq \lambda^{p} \int_{\left[E \left[V^{\left.\left.-1 /(p-1) \mid F_{n}\right]>\lambda^{p /(p-1)}\right\}}\right.\right.} U d P \leqq W_{p} .
$$

Letting $\lambda \rightarrow \infty$, we have $U=0$ a.s. on $B_{\infty}$. Thus

$$
\Omega=\left(\underset{\substack{-\infty<i<\infty \\-\infty<j<\infty}}{\bigcup} B_{i j}\right) \cup\left\{\omega: E\left[U \mid F_{n}\right]=0 \text { or } E\left[V^{-1 /(p-1)} \mid F_{n}\right]=0\right\} .
$$

Therefore

$$
\left(\alpha^{-1} E\left[V^{-1 /(p-1)} \mid F_{n}\right]\right)^{p-1}\left(\alpha^{-1} E\left[U \mid F_{n}\right]\right) \leqq W_{p} \alpha \quad \text { a.s. on } \Omega .
$$

By (1) and the arbitrariness of $\alpha(>1)$ and $n$, we get

$$
A_{p}=W_{p} \quad \text { for } \quad p \in(1, \infty) .
$$

Since

$$
\begin{gathered}
E\left[V^{-1 /(p-1)} \mid F_{n}\right] E\left[U \mid F_{n}\right]^{1 /(p-1)}=E\left[\left(V^{-1} E\left[U \mid F_{n}\right]\right)^{1 /(p-1)} \mid F_{n}\right] \leqq A_{1}^{1 /(p-1)}, \\
\lim _{p \downarrow 1} A_{p} \leqq A_{1} .
\end{gathered}
$$

On the other hand, by Lemma 2

$$
V^{-1} E\left[U \mid F_{n}\right] \leqq \lim _{m \rightarrow \infty} E\left[V^{-m} \mid F_{n}\right]^{1 / m} E\left[U \mid F_{n}\right] .
$$

Thus we get $A_{1}=\lim _{p \downarrow 1} A_{p}$. Then by Lemma 3 and (2) we get $A_{1}=W_{1}$.

2. The reverse Hölder inequality. Let $(\Omega, F, P), \quad F_{1} \subset F_{2} \subset \cdots \subset$ $F_{n} \subset \cdots \subset F$ and $V$ be as in Section 1. In this section further we assume $F_{1}=\{\varnothing, \Omega\}$. For $p \in(1, \infty)$ set

$$
A_{p}(V)=\sup _{n}\left\|E\left[V^{-1 /(p-1)} \mid F_{n}\right]^{p-1} E\left[V \mid F_{n}\right]\right\|_{\infty}
$$


and

$$
H_{\delta}(V)=\sup _{n}\left\|E\left[V^{1+\delta} \mid F_{n}\right]^{1 /(1+\delta)} E\left[V \mid F_{n}\right]^{-1}\right\|_{\infty} .
$$

Recently C. Watari has pointed out the following

THEOREM C. Let $\left(F_{n}\right)$ be regular, that is, each $F_{n}$ is atomic and there is a constant $C_{0}>0$ satisfying $P(B) / P(D)<C_{0}$ for any two atoms $B \in F_{n-1}$ and $D \in F_{n}$ with $B \supset D$. Then for each $p \in(1, \infty)$ and $M \in(1, \infty)$ there exist $\delta\left(p, M, C_{0}\right)>0$ and $N\left(p, M, C_{0}\right)<\infty$ such that $H_{\delta}(V) \leqq N$ provided that $A_{p}(V) \leqq M$.

Now we show that the regularity of $\left(F_{n}\right)$ in the above theorem is necessary.

Theorem 2. Assume that there exist $M, N>1, p \in(1, \infty)$ and $\delta>0$ such that $H_{\delta}(V) \leqq N$ provided that $A_{p}(V) \leqq M$. Then $\left(F_{n}\right)$ is regular.

Proof. Assume that $F_{n}$ is atomic. Let $B$ be an arbitrary atom of $F_{n}$. Let $D \in F_{n+1}$ and $D \subset B$. Set

$$
V=1+(M-1) P(B) I(D) / P(D) .
$$

Then

$$
E\left[V \mid F_{m}\right] E\left[V^{-1 /(p-1)} \mid F_{m}\right]^{p-1}=1 \quad \text { for } \quad m \geqq n+1
$$

and

$E\left[V \mid F_{m}\right] E\left[V^{-1 /(p-1)} \mid F_{m}\right]^{p-1} \leqq M \quad$ for $\quad m \leqq n, \quad$ i.e., $\quad A_{p}(V) \leqq M$.

Since

$$
E\left[V^{1+\delta} \mid F_{n}\right] \geqq(M-1)^{1+\delta}(P(B) / P(D))^{\delta} \quad \text { on } B \text { and } \quad E\left[V \mid F_{n}\right] \leqq M,
$$

by hypothesis

$$
(P(B) / P(D))^{\delta /(1+\delta)} \leqq N M(M-1)^{-1} .
$$

Thus $F_{n+1}$ is also atomic and (3) is satisfied for any two atoms $B \in F_{n}$ and $D \in F_{n+1}$ with $D \subset B$, that is, $\left(F_{n}\right)$ is regular.

Finally we add the following

THEOREM 3. If $\left(F_{n}\right)$ is not regular, there exists $V$ such that $A_{1}(V)<\infty$ and $H_{\delta}(V)=\infty$ for any $\delta>0$, where

$$
A_{1}(V)=\sup _{n}\left\|V^{-1} E\left[V \mid F_{n}\right]\right\|_{\infty} \text {. }
$$

Proof. First assume that $F_{n}$ does not consist of a finite number of atoms for some $n$. Since $F_{1}=\{\varnothing, \Omega\}$, we may assume $F_{n-1}$ consists of a finite number of atoms. Let $D_{k} \in F_{n}, D_{k} \subset B, \quad 0<P\left(D_{k}\right) / P(B)<2^{-k^{2}}$ 
for $k=1,2, \cdots, D_{k} \cap D_{h}=\varnothing(k \neq h)$ and $B$ be an atom of $F_{n-1}$. Set

$$
V=1+\sum_{k=1}^{\infty} 2^{-k} P(B) I\left(D_{k}\right) / P\left(D_{k}\right) \text {. }
$$

Then $A_{1}(V) \leqq 2$ and

$$
E\left[V^{1+\delta} \mid F_{n}\right] \geqq \sum_{k=1}^{\infty} 2^{-k(1+\delta)}\left(P(B) / P\left(D_{k}\right)\right)^{\delta}=\infty
$$

on $B$ for any $\delta>0$. So, $H_{\delta}(V)=\infty$ for any $\delta>0$.

Thus we may assume $F_{n}$ consists of a finite number of atoms. Let $\left\{B_{n}\right\}$ and $\left\{D_{n}\right\}$ be sequences of atoms of $\left\{F_{i(n)}\right\}$ and $\left\{F_{i(n)+1}\right\}$ respectively such that $D_{n} \subset B_{n}, \lim P\left(D_{n}\right) / P\left(B_{n}\right)=0$ and $P\left(D_{n}\right)<\min \left(2^{-n^{2}}, 4^{-1} P\left(D_{n-1}\right)\right)$.

Assume that inf $P\left(B_{n}\right)=c_{0}>0$. Set

$$
V=1+\sum_{k=1}^{\infty} 2^{-k} P\left(D_{k}\right)^{-1} I\left(D_{k} \cap\left(\bigcap_{h>k} D_{h}^{c}\right)\right) .
$$

Take an arbitrary $n$ and an arbitrary atom $B$ of $F_{n}$. If $P(B) \geqq c_{0}$, then $E\left[V \mid F_{n}\right] \leqq 2 c_{0}^{-1}$ on $B$. If $P(B)<c_{0}$, then $E\left[V \mid F_{n}\right]=V$ on $B$. Thus $A_{1}(V) \leqq 2 c_{0}^{-1}$. On the other hand,

$$
E\left[V^{1+\delta} \mid F_{1}\right] \geqq \sum 2^{-k(1+\delta)} P\left(D_{k}\right)^{-1-\delta} P\left(D_{k}\right) / 2=\infty,
$$

so $H_{\delta}(V)=\infty$ for any $\delta>0$.

Assume that $\lim \inf _{n \rightarrow \infty} P\left(B_{n}\right)=0$. In this case we may assume $i(n)+1<i(n+1)$ and

$$
P\left(D_{n}\right)>2 P\left(B_{n+1}\right) \text {. }
$$

By selecting a subsequence, if necessary, it suffices to consider the following two cases:

$$
\begin{gathered}
B_{1} \supset B_{2} \supset \cdots, \\
B_{h} \cap B_{k}=\varnothing \quad(h \neq k) .
\end{gathered}
$$

In the case of (6), set

$$
V=1+\sum_{k=1}^{\infty} P\left(B_{k}\right) I\left(D_{k}\right) / P\left(D_{k}\right) .
$$

Then $A_{1}(V) \leqq 2$ and

$$
E\left[V^{1+\delta} \mid F_{i(n)}\right]^{1 /(1+\delta)} E\left[V \mid F_{i(n)}\right]^{-1} \geqq\left(P\left(B_{n}\right) / P\left(D_{n}\right)\right)^{\delta /(1+\delta)} 2^{-1}
$$

on $B_{n}$. Thus $H_{\delta}(V)=\infty$ for any $\delta>0$.

Lastly in the case of (5), we define $V$ and $\left\{\alpha_{k}\right\}_{k=1}^{\infty}$ as follows. Let $V=1$ on $B_{1}^{c}$ and $\alpha_{1}=1$. When $V$ is defined on $B_{k}^{c}$ and $\alpha_{k}$ is defined, let 


$$
\begin{gathered}
V=\alpha_{k} \quad \text { on } B_{k} \cap D_{k}^{c} \cap B_{k+1}^{c}, \\
V=\alpha_{k} P\left(B_{k}\right) / P\left(D_{k}\right) \text { on } D_{k} \cap B_{k+1}^{c}, \\
\alpha_{k+1}=\alpha_{k} \quad \text { if } B_{k+1} \not \subset D_{k}, \\
\alpha_{k+1}=\alpha_{k} P\left(B_{k}\right) / P\left(D_{k}\right) \quad \text { if } B_{k+1} \subset D_{k} .
\end{gathered}
$$

Then

$$
\int_{B_{k} \backslash B_{k+1}} V d P \leqq 2 \alpha_{k} P\left(B_{k}\right)
$$

and

$$
\alpha_{j} \leqq \alpha_{k} \prod_{i=k}^{j-1}\left(P\left(B_{i}\right) / P\left(D_{i}\right)\right) .
$$

Assume that $i(k-1)+1 \leqq n \leqq i(k)$ and $B$ is an arbitrary atom of $F_{n}$. If $B \not \supset B_{k}, E\left[V \mid F_{n}\right]=V$ on $B$. If $B \supset B_{k}$, by (4), (7), and (8)

$$
\begin{aligned}
& E\left[V \mid F_{n}\right]=P(B)^{-1} \int_{B} V d P \leqq P(B)^{-1}\left\{\alpha_{k} P(B)+\sum_{j=k}^{\infty} 2 \alpha_{j} P\left(B_{j}\right)\right\} \\
& \quad \leqq 2 P(B)^{-1}\left\{\alpha_{k} P(B)+\sum_{j=k}^{\infty} \alpha_{k}\left(P\left(B_{k}\right) / P\left(D_{k}\right)\right) \cdots\left(P\left(B_{j-1}\right) / P\left(D_{j-1}\right)\right) P\left(B_{j}\right)\right\} \leqq 6 \alpha_{k}
\end{aligned}
$$

and $V^{-1} \leqq \alpha_{k}^{-1}$ on $B$. Thus $A_{1}(V) \leqq 6$. But on $B_{k}$

$$
E\left[V^{1+\delta} \mid F_{i(k)}\right] \geqq \alpha_{k}^{1+\delta}\left(P\left(B_{k}\right) / P\left(D_{k}\right)\right)^{\delta} / 2
$$

and $E\left[V \mid F_{i(k)}\right] \leqq 4 \alpha_{k}$. Thus $H_{\delta}(V)=\infty$ for any $\delta>0$.

\section{REFERENCES}

[1] R. Corfman and C. Fefrerman, Weighted Norm Inequalities for Maximal Functions and Singular Integrals, Studia Math., 51 (1974), 241-250.

[2] M. IzUMISAWA AND N. KazAMAKI, Weighted Norm Inequalities for Martingales, Tôhoku Math. J., 29 (1977), 115-124.

[3] B. Muckenhoupt, Weighted norm inequalities for the Hardy maximal function, Trans. Amer. Math. Soc., 165 (1972), 207-226.

DepartMent of MATHEMatics

College of General Education

TÔHOKU UNIVERSITY

SENDAI, 980 JAPAN 